\title{
Force Correlations, Anisotropy, and Friction Mobilization in Granular Assemblies under Uniaxial Deformation.
}

\author{
O. I. Imole, M. Wojtkowski, V. Magnanimo and S. Luding \\ Multi Scale Mechanics (MSM), Faculty of Engineering Technology, MESA+, \\ University of Twente, P.O. Box 217, 7500 AE Enschede, The Netherlands
}

\begin{abstract}
We study dense, frictional, polydisperse 3D granular assemblies under uniaxial deformation with Discrete Element Method (DEM) simulations. The overall goal - beyond the scope of the present study - is to link microscopic parameters and observations with the macroscopic behavior, for different elementary deformation modes.

At present, we focus on the behavior of the force/contact network during uniaxial deformation, for different coefficients of friction. We discuss the stress and structural anisotropy and the relationship between force intensity weighted by contact state (sticking or sliding, at the Coulomb limit) or force strength. Furthermore, we study the orientational distribution of contacts and forces and the contribution of friction to structural anisotropy. We find that initial isotropic states are irrecoverable, since the structural anisotropy is independent of the deviatoric stress behavior both with and without friction. Contacts display an interesting anisotropy of order four in the presence of friction.
\end{abstract}

Keywords: DEM, Friction, Sliding Contacts, Uniaxial Deformation, PARDEM PACS: $45.70 . \mathrm{Cc}, 81.05 . \mathrm{Rm}, 81.20 . \mathrm{Ev}$

\section{INTRODUCTION}

Granular materials are omnipresent in nature and widely used in various industries ranging from food, pharmaceutical, agriculture and mining - among others. In many granular systems, interesting phenomena like dilatancy, anisotropy, shear-band localization, history-dependence, jamming and yield have attracted significant scientific interest over the past decade [1]. The bulk behavior of these materials depends on the behavior of their constituents (particles) interacting through contact forces. To understand the deformation behavior of these materials, various laboratory element tests can be performed [2, 3]. Element tests are (ideally homogeneous) macroscopic tests in which one can control the stress and/or strain path. Such macroscopic experiments are important ingredients in developing constitutive relations, but they provide little information on the microscopic origin of the bulk flow behavior. As an alternative, the Discrete Element Method (DEM) [4] - which provides information about the micro-structure beyond what is experimentally accessible - can be used. In this study, we focus on one specific element test, namely uniaxial deformation.

Besides the contact network and the probability distribution of normal contact forces, another interesting issue is the distribution and orientation of contacts during the deformation of dense frictional packings [5, 6, 7]. In early, two-dimensional studies on frictional avalanching [5], it has been observed that the friction is mobilized mostly from weak contacts, whereas strong contacts resist friction mobilization.
The final goal is to investigate and understand the dependencies between the microscopic observations presented hereafter and the evolution of macroscopic quantities as pressure and deviatoric stress - and to further extend this to explain the evolution of the structural/contact and force/stress anisotropies. We first describe the simulation method and model parameters before both the stress and structure anisotropies are reported. Where given, anisotropy refers to not only the deviatoric stress, but also to the direction-dependence and inhomogeneity of forces, i.e., its microscopic origin. Then we classify the sliding/non-sliding contacts at the Coulomb limit according to their normal force, and finally examine the polar orientation of the contacts.

\section{SIMULATION PROCEDURE}

We use the Discrete Element Method (DEM) [4] with a simple linear visco-elastic normal contact force law $f^{n} \hat{\mathbf{n}}=(k \delta+\gamma \dot{\boldsymbol{\delta}}) \hat{\mathbf{n}}$, where $k$ is the spring stiffness, $\gamma$ is the contact viscosity parameter and $\delta$ or $\dot{\delta}$ are the overlap or the relative velocity in the normal direction $\hat{\mathbf{n}}$. The normal force is complemented by a tangential force law [4], such that the total force at contact $c$ is: $\mathbf{f}_{c}=f_{n} \hat{\mathbf{n}}+f_{t} \hat{\mathbf{t}}$, where $\hat{\mathbf{n}} \cdot \hat{\mathbf{t}}=0$. A summary of the values of the parameters used is shown in Table 1, with sliding and sticking friction $\mu=\mu_{s l}=\mu_{s t}$ and rolling- and torsion-torques inactive $\left(\mu_{r}=\mu_{t}=0\right)$. An artificial viscous dissipation force proportional to the velocity of the particle is added for both translational and rotational degrees of freedom, 
TABLE 1. Summary and numerical values of particle parameters used in the DEM simulations, where $\mu=0,0.01$, and 0.1 are presented here; for details see Ref. [4].

\begin{tabular}{lrll}
\hline & Value & Unit & Description \\
\hline$N$ & 9261 & {$[-]$} & Number of particles \\
$\langle r\rangle$ & 1 & {$[\mathrm{~mm}]$} & Average radius \\
$w$ & 1.5 & {$[-]$} & Polydispersity $w=r_{\max } / r_{\min }$ \\
$\rho$ & 2000 & {$\left[\mathrm{~kg} / \mathrm{m}^{3}\right]$} & Particle density \\
$k_{n}$ & $10^{5}$ & {$\left[\mathrm{~kg} / \mathrm{s}^{2}\right]$} & Normal spring stiffness \\
$k_{t}$ & $2.10^{4}$ & {$\left[\mathrm{~kg} / \mathrm{s}^{2}\right]$} & Tangential spring stiffness \\
$\mu$ & vary & {$[-]$} & Coefficient of friction \\
$\gamma_{n}$ & 100 & {$[\mathrm{~kg} / \mathrm{s}]$} & Viscosity-normal direction \\
$\gamma_{t}$ & 20 & {$[\mathrm{~kg} / \mathrm{s}]$} & Viscosity -tang. direction \\
$\gamma_{b t}$ & 100 & {$[\mathrm{~kg} / \mathrm{s}]$} & Background damping-trans. \\
$\gamma_{b r}$ & 20 & {$[\mathrm{~kg} / \mathrm{s}]$} & Background damping-rotational \\
$\tau_{c}$ & 0.64 & {$[\mu \mathrm{s}]$} & Contact duration (average) \\
\hline
\end{tabular}

resembling the damping due to a background medium, as e.g. a fluid.

The simulation set-up is a cuboid volume [8], triaxial box, with periodic boundaries on all sides. Since careful, well-defined sample preparation is essential to obtain reproducible results [9], we follow a three-step procedure: (i) Spherical particles are randomly generated in the 3D box with low volume fraction and rather large random velocities, such that they have sufficient space and time to exchange places and to randomize themselves. (ii) This granular gas is then isotropically compressed to a target volume fraction $v_{0}$. The goal is to approach a direction independent, isotropic configuration slightly above the jamming volume fraction $v_{c}$, i.e. the transition point from fluid-like behavior to solid-like behavior [10]. (iii) This is followed by a relaxation period at constant volume fraction to allow the particles to dissipate their kinetic energy and to achieve a static configuration in mechanical equilibrium.

As element test, the uniaxial compression is then achieved by moving the periodic walls in the $z$-direction according to a prescribed co-sinusoidal strain path [8], with diagonal strain-rate tensor $\dot{\varepsilon}_{u}(0,0,-1)$, where positive $\dot{\varepsilon}_{z z}$ denotes compression - while the other boundaries $x$ and $y$ are non-mobile. During loading (compression) the volume fraction increases from $v_{0}$ (at dimensionless time $\tau=0)$ to a maximum $v_{\max }=0.820(\tau=0.5)$ and reverses back to the original $v_{0}$ at the end of the cycle $(\tau=1)$, after complete unloading. For more details on preparation and other parameters, see Ref. [8].

\section{RESULTS}

Stress and Structure Anisotropy: Under uniaxial compression, not only does shear stress build up, but also the

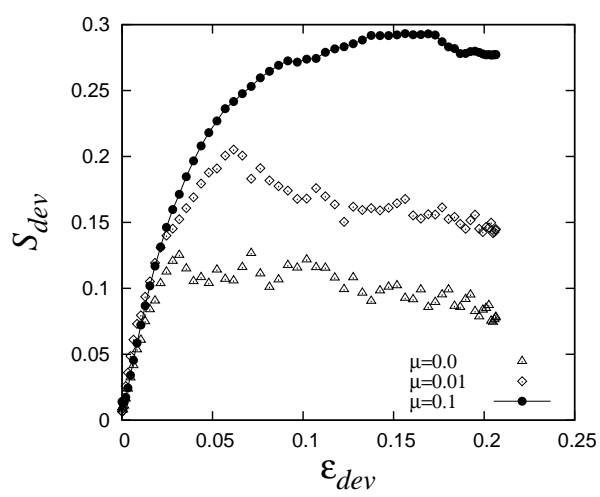

FIGURE 1. Deviatoric stress ratio $\left(S_{d e v}=\sigma_{d e v} / p\right)$ plotted against deviatoric strain for uniaxial compression with $\mu$ given.

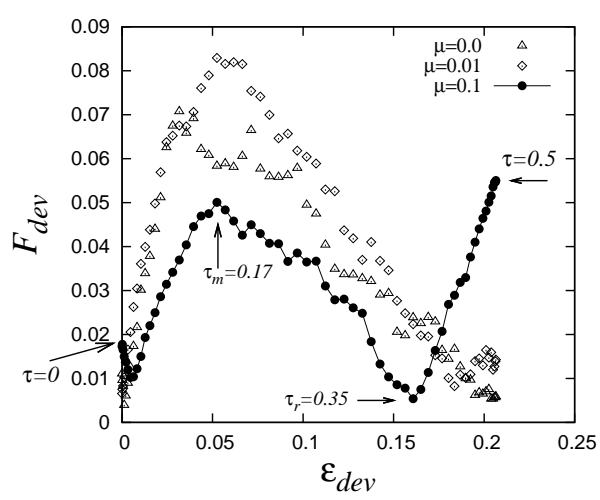

FIGURE 2. Deviatoric fabric plotted against deviatoric strain from the same simulations as in Fig. 1. Only the loading half-cycle from $\tau=0$ to $\tau=0.5$ is shown.

anisotropy of the contact and force networks develops, as it relates to the creation and destruction of new contacts [8]. We term the deviatoric part of the stress tensor the stress anisotropy, in parallel to the direction dependency of the structural anisotropy. The deviatoric stress ratio, $S_{d e v}=\sigma_{d e v} / p$, quantifies the stress anisotropy as shown in Fig. 1 for a frictionless $(\mu=0)$ and frictional $(\mu=0.01,0.1)$ systems under uniaxial loading - where $p$ is the pressure, and $\sigma_{d e v}=\sqrt{3 J_{2}^{\sigma}}$ is the deviatoric stress, with $J_{2}^{\sigma}$ the second invariant of the deviatoric stress tensor. The stress anisotropy initially grows with applied strain until a maximum is reached, at different strains, from where it decreases slightly. This "macroscopic friction coefficient", $\mu^{\text {macro }}:=S_{d e v}$ represents the mobilized "friction", i.e. shear resistance, along the loading path and is higher than the microscopic coefficients of contact friction - at least for the parameters used here [8].

Along with the stress, we introduce the fabric tensor as defined in Ref. [8], in order to fully characterize the contact network of the aggregate. The deviatoric fabric mag- 
nitude, $F_{d e v}=\sqrt{3 J_{2}^{F}}$, (see Ref. [8]), quantifies the structural anisotropy, as shown in Fig. 2. It builds up from different (random, but small) initial values and reaches different maxima at the same level of $\approx 5 \%$ deviatoric strain $\left(\tau_{m}=0.17\right)$. For larger strain, the structural anisotropy is decreasing rapidly towards zero. Interestingly, for the largest $\mu=0.1$, starting from $\tau_{r}=0.35$, further loading in the axial direction leads to an increase of the deviatoric fabric, until at maximum compression $(\tau=0.5)$, the deviatoric fabric again reaches a local maximum. This indicates that more contacts are created in the axial direction compared to the horizontal plane at the beginning of the loading cycle. At $\tau_{m}$ however, the material behavior changes such that the rate of contact creation in the $x-y$ plane becomes higher. The micromechanical origin of this surprising behavior is the motivation for the current study and will be reported in more detail elsewhere [11]. Unloading the system back to its initial volume fraction up to time $\tau=1$ (not shown), the initial state is not recovered - a clear signature of history dependence and structural anisotropy being independent of (or decoupled from) the deviatoric stress behavior.

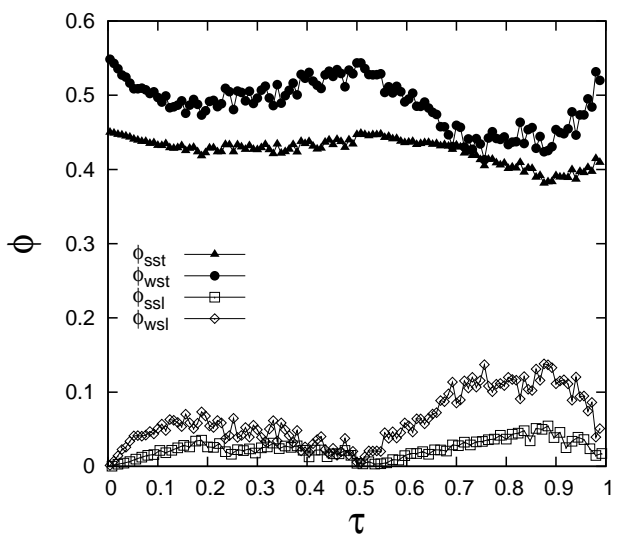

FIGURE 3. Fraction of weak sliding $\left(\phi_{w s l}\right)$, strong sliding $\left(\phi_{s s l}\right)$, weak sticking $\left(\phi_{w s t}\right)$ and strong sticking $\left(\phi_{s s t}\right)$ contacts, during a uniaxial loading- and unloading-cycle, with $\mu=0.1$.

Friction Mobilization: Mobilization of contact friction, during uniaxial deformation of the bulk material, occurs when $f_{t} / \mu f_{n} \rightarrow 1$. The tangential forces grow towards their limit and support larger shear stress; for tangential forces at/above the Coulomb limit, i.e., at fully mobilized friction, sliding sets in and rearrangements of contacts can lead to new, more stable configurations. It has been observed [12] that sliding is mostly active at weak contacts, while stronger contacts stay in the sticking regime and sustain larger friction forces [12] but are less mobilized. Weak and strong contacts are defined relative to the average normal force; $f^{*}=f_{n} /\left\langle f_{n}\right\rangle<1$ are termed weak and $f^{*}>1$ are termed strong [12], with dominating sliding and sticking, respectively. We find that this friction mobilization rule is not strictly true in the case $\mu=0.1$, as there may be a considerable weak contacts with friction not fully mobilized (termed weak sticking), as well as strong contacts fully mobilized (termed strong sliding).

In Fig. 3, the fractions of strong and weak, sliding and sticking contacts are plotted against time, where the $\phi$ are defined as $C / C_{t o t}$ where $C$ is the number of contacts in the category and $C_{t o t}$ is the total number of contacts. From this data, it is evident that most contacts are sticking - with very few sliding contacts - irrespective of the contact type. Interestingly, sticking (solid symbols) and sliding (open symbols) contacts correlate well with each other regardless of intensity (weak or strong). All data are non-symmetric around stress reversal at $\tau=0.5$. Focusing on the sliding contacts, the weak sliding contacts increase sharply compared to the stronger sliding contacts at the beginning of loading and after strain reversal. As compression progresses, the fraction of sliding contacts decays at $\tau=0.5$ and $\tau=1$. Interestingly, a stronger increase in the fraction of sliding contacts is also seen at the beginning of the unloading branch compared to the loading branch.

The fractions of strong and weak sticking contacts both decay at the beginning of the compression cycle, followed by an increase as compression progresses. At maximum compression $(\tau=0.5)$ almost all contacts are weak sticking with $\approx 54$ percent of the total contacts weak sticking compared to $\approx 46$ percent for contacts with stronger forces that do not lead to complete friction mobilization (strong sticking). From these maxima at $\tau=0.5$, the fractions decrease and sharply increase just before the end of unloading $(\tau=1)$.

A reduction of $\mu$ leads to a significant increase in fluctuations (data not shown). This is accompanied by an increase in the total fraction of sliding contacts and a considerable decrease in the fraction of sticking forces.

Polar Representation: To better understand the orientation and arrangement of the contacts, we present the polar representation of contacts, forces and the mobilized friction. Given the three normal unit vector components $\hat{n}_{x}, \hat{n}_{y}$, and $\hat{n}_{z}$ for each contact pair, one needs the orientation $\theta$ of the normal unit vector in the direction relative to the active (axial) direction. To obtain this, we average over the spherical azimuthal (vs. polar) $(r, \varphi)$ coordinate and calculate the polar angle as $\arccos \left(\hat{n}_{z}\right)$. We then divide the vectors, based on their orientation into bins with width $\Delta \theta=10^{\circ}$. The fraction of contacts in a single bin is defined as $\phi^{\theta}=C_{t o t}^{\theta} / C_{t o t}$, where $C_{t o t}^{\theta}=\sum_{C \in b} 1$ and $b \in[\theta-\Delta \theta / 2 ; \theta+\Delta \theta / 2]$. Furthermore, $\phi^{\theta}$ is normalized with the surface of the spherical annulus for each $\Delta \theta$ by the factor $\Delta \theta \sin \theta$ to yield the azimuthal contact probability density $P(\theta)=\left(\phi^{\theta} / \Delta \theta \sin \theta\right)$ such that $\int_{0}^{\pi} P(\theta) \sin \theta \Delta \theta=1$. The polar distribution of the normal forces and the mobilized friction are given respectively, by $\left(\sum_{C \in b} f_{n}\right) /\left(C_{\text {tot }}^{\theta}\right)$ and $\left(\sum_{C \in b}\left(f_{t} / \mu f_{n}\right)\right) /\left(C_{\text {tot }}^{\theta}\right)$, 


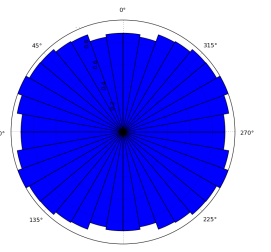

$\tau=0.5, C$

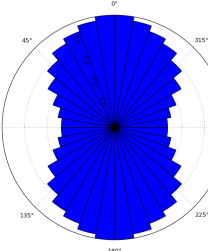

$\tau=0.5, f_{n}$

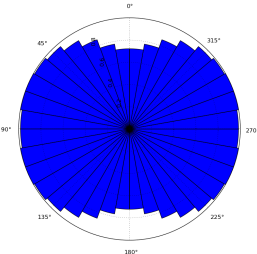

$\tau=0.5, f_{t} / \mu f_{n}$
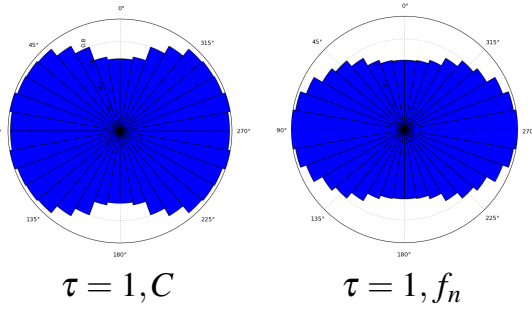

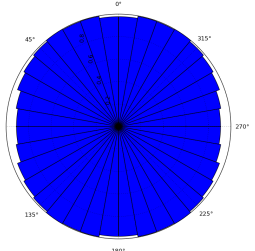

$\tau=1, f_{t} / \mu f_{n}$

FIGURE 4. Polar distribution of the contacts $C$, normal force $f_{n}$ and mobilized friction $f_{t} / \mu f_{n}$ for $\mu=0.1$, at maximum compression $(\tau=0.5)$ and at the end of the decompression cycle $(\tau=1)$. The inital states are not shown since they are isotropic, and the vertical corresponds to the active axial compression/tension direction, $\theta=0^{\circ}$.

where the normalization with the number of contacts in each bin has been introduced.

Fig. 4 shows the polar distribution of contacts $C$, normal forces $f_{n}$, along with the distribution of the microscopic mobilized friction $f_{t} / \mu f_{n}$ at different steps along the uniaxial compression. Note that each polar representation is scaled by its maximum, so that only the shapes can be compared - not the magnitudes of the polar plots. The initial state (not shown) for the three parameters is almost isotropic with contacts, forces and mobilized friction evenly distributed along the polar surface. As compression begins, anisotropy develops and more contacts align along the main compressive vertical direction $\theta=0^{\circ}$. Interestingly, at $\tau=0.5$, while the stronger contacts are still aligned vertically, many more contacts appeared in the horizontal plane, in agreement with the behavior of the deviatoric fabric in Fig. 2. During unloading, a reorganization of the force network occurs so that both the stronger forces and the contacts align horizontally. At the end of the decompression cycle $(\tau=1)$, the initial (isotropic) state is not recovered, both for contacts and normal forces.

The polar distribution of $f_{t} / \mu f_{n}$ is even more interesting. Starting from an isotropic configuration at $\tau=0$ (not shown), a change in the polar distribution can be seen at maximum compression $(\tau=0.5)$. At this point, friction is mostly fully mobilized along the direction perpendicular to the axial (preferential direction of $f_{n}$ ). At the end of the unloading branch, the system is restored close to (but not exactly) its initial isotropic mobilization. For lower friction, the resistance to lateral dilation is reduced such that the observations concerning the distribution of $f_{n}$ and $f_{t} / \mu f_{n}$ are less/more pronounced, respectively (data not shown, see Ref. [11]).

\section{SUMMARY AND OUTLOOK}

In this paper, we have used the discrete element method to investigate the microscopic and macroscopic behavior of frictional assemblies under uniaxial deformation. Sliding and sticking contact evolution - regardless of inten- sity - correlate well with few sliding and many sticking contacts, for both weak and strong forces. The irrecoverability of the initial system state at the end of a full cycle shows the independence of the structural anisotropy on the deviatoric stress behavior.

Further studies will focus on understanding the interesting reversal in structural anisotropy during loading and a description of the polar representations for contacts and forces by a low order harmonic approximation of a Fourier expansion. Also the effects of different contact models [4] on the findings will be investigated.

Acknowledgements: Helpful discussions with V. Ogarko, M. Ramaioli, N. Kumar, E. Chavez J. Ooi and F. Radjai are acknowledged. This work is financially supported by the European Union funded Marie Curie Initial Training Network, PARDEM, FP7 (ITN-238577), see http://www.pardem.eu/ for more information.

\section{REFERENCES}

1. M. Nakagawa, and S. Luding, editors, Powders and Grains 2009, AIP, Golden, Colorado, USA, 2009.

2. J. Schwedes, Granular Matter 5, 1-43 (2003).

3. G. D. R. MiDi, The European Physical Journal E: Soft Matter and Biological Physics 14, 341-365 (2004).

4. S. Luding, Granular Matter 10, 235-246 (2008).

5. F. Radjai, S. Roux, and J. J. Moreau, Chaos 9, 544-550 (1999).

6. A. S. J. Suiker, and N. A. Fleck, Journal of Applied Mechanics 71, 350-358 (2004).

7. C. Thornton, and S. J. Anthony, Philosophical Transactions of the Royal Society of London. Series A: Mathematical, Physical and Engineering Sciences 356, 2763-2782 (1998).

8. O. I. Imole, N. Kumar, V. Magnanimo, and S. Luding, KONA Powder and Particle Journal 30, 84-108 (2013).

9. A. Ezaoui, and H. Di Benedetto, Géotechnique 59, 621-635 (2009).

10. M. van Hecke, Journal of Physics: Condensed Matter 22, 033101 (2009).

11. O. I. Imole, M. B. Wojtkowski, N. Kumar, V. Magnanimo, and S. Luding, In preparation (2013).

12. L. Staron, and F. Radjai, Physical Review E 72, 041308:15 (2005). 